\title{
Características celulares e bioquímicas do líquido peritoneal de equinos submetidos à obstrução experimental do duodeno, íleo e cólon maior
}

\author{
[Cellular and biochemical characteristics of peritoneal fluid of equines submitted to experimental \\ obstruction of duodenum, ileum, and large colon] \\ P.A. Di Filippo, A.E. Santana, A.F.S. Nogueira, L.A. Anai, E. Campos Filho \\ Faculdade de Ciências Agrárias e Veterinárias - UNESP \\ Via de Acesso Prof. Paulo Donato Castellane, s/n \\ 14884-900 - Jaboticabal, SP
}

\begin{abstract}
RESUMO
Estudaram-se as características macroscópicas, bioquímicas e citológicas do líquido peritoneal de equinos submetidos a um modelo experimental de obstrução intestinal em 24 animais, distribuídos em grupos: de controle instrumentado (GI), de obstrução do duodeno (GII), de obstrução do íleo (GIII) e de obstrução do cólon maior (GIV). As amostras de líquido peritoneal foram colhidas antes da cirurgia (T0), durante as obstruções (T60i-T180i) e após as desobstruções (T60ri-T7ํㅜ). Durante o período obstrutivo, não foram observadas alterações significativas nos parâmetros bioquímicos e citológicos avaliados no líquido peritoneal dos animais. Após as desobstruções, apenas os animais de GII e GIII apresentaram resposta inflamatória mais intensa, caracterizada por maior contagem global e diferencial de leucócitos e dos valores de proteína total, fibrinogênio, lactato e fósforo inorgânico. As alterações laboratoriais não foram associadas a sinais clínicos indicativos da presença de processo inflamatório abdominal, demonstrando que os resultados da análise do líquido peritoneal, apesar de auxiliarem no acompanhamento da evolução do processo de cura, não devem ser utilizados isoladamente na elaboração do diagnóstico e do prognóstico de complicações no pós-operatório de equinos com cólica.
\end{abstract}

Palavras-chave: cavalo, fluido peritoneal, cólica, peritonite

\begin{abstract}
The macroscopic, biochemical, and cytological characteristics of peritoneal fluid of equines submitted to intestinal obstruction using an experimental model were evaluated. Twenty-four animals were distributed in four groups: instrumented control (GI), duodenum obstruction (GII), ileum obstruction (GIII), and large colon obstruction (GIV). Peritoneal fluid samples were collected before the surgery (T0), during the obstruction (T60i-T180i), and after unblocking procedures (T60ri-T7 ${ }^{\circ}$ ). During obstructive period, significant alterations were not observed in biochemical and cytological examination of peritoneal fluid of all animals. After unblocking procedure, animals from GII and GIII presented intense inflammatory response characterized by higher global and differential leukocytes counts, as well as in fibrinogen and total protein concentrations; lactate and inorganic phosphorus concentrations in peritoneal fluid were also increased. The laboratory alterations were not associated with clinical signs, indicative of the abdominal inflammatory process presence. Results showed that analyses of peritoneal fluids can be used as support in the evolution of healing process. However, they can not be isolated used for a diagnosis and prognosis of equine postoperative complications.
\end{abstract}

Keywords: equine, peritoneal fluid, colic, peritonitis

\section{INTRODUÇÃO}

A síndrome cólica é uma das urgências mais frequentes na clínica de equinos, e as alterações ocorridas nas alças intestinais resultam em importantes alterações clínicas e laboratoriais (Thomassian, 1996).

O exame do líquido peritoneal é considerado o teste laboratorial mais esclarecedor para o

Recebido em 21 de maio de 2009

Aceito em 25 de novembro de 2009

E-mail: paula_difilippo@yahoo.com.br 
estabelecimento do diagnóstico, para o direcionamento da terapia e também para determinar a severidade da lesão abdominal (Tulleners, 1983). A inflamação do peritônio ou peritonite pode ocorrer em resposta a uma variedade de estímulos tanto infecciosos (bactérias, vírus, fungos e parasitas) quanto não infecciosos (traumas, agentes químicos e neoplasias). Independentemente da causa, a inflamação do peritônio provoca sensíveis alterações no exame do líquido peritoneal. O peritônio inflamado apresenta permeabilidade aumentada, o que resulta em aumento do volume do fluido peritoneal e da concentração de proteínas. A liberação local de substâncias quimiotáxicas promove a migração de leucócitos para a cavidade peritoneal. O número de hemácias também pode estar aumentado, quando a lesão for muito severa (Ricketts 1987; Mendes et al., 1999a).

O presente estudo teve os objetivos de avaliar e comparar as alterações citológicas e bioquímicas no líquido peritoneal de equinos submetidos à obstrução experimental do duodeno, do íleo e do cólon maior, determinando o início e o comportamento, em função do tempo, de tais alterações, e se essas podem ser utilizadas no diagnóstico e no prognóstico de obstruções intestinais específicas em equinos com cólica.

\section{MATERIAL E MÉTODOS}

Pesquisa aprovada pela Comissão de Ética e Bem Estar Animal (CEBEA), protocolo $\mathrm{n}^{\circ}$ 023232-05.

Utilizaram-se 24 equinos, oito fêmeas (não gestantes), 16 machos (12 castrados e quatro não castrados), sem raça definida, com idades entre dois e 15 anos, escore corporal de três a quatro e peso corporal médio de $295,9 \mathrm{~kg}$. Uma semana antes do experimento, após avaliação clínica, fez-se o controle de endoparasitas (mebendazol ${ }^{1}$, $50 \mathrm{mg} \mathrm{kg}^{-1}$ ) e de ectoparasitas (deltametrina ${ }^{2}$ a $0,025 \%)$. Os animais foram colocados em piquetes coletivos sob dieta à base de feno de coast cross (Cynodon dactylon) e água à vontade. A ração concentrada comercial $^{3}$ foi

${ }^{1}$ Platelmin Equino - UCB S/A - São Paulo, Brasil.

${ }^{2}$ Butox P - Intervet S/A - São Paulo, Brasil.

${ }^{3}$ Tec Horse - Purina - Paulínea, Brasil. fornecida duas vezes ao dia, em quantidade equivalente a $1 \%$ do peso corpóreo $(2,5$ a $3,4 \mathrm{~kg})$, adicionada de $50 \mathrm{~g} \mathrm{dia}^{-1}$ de suplemento mineral ${ }^{4}$.

Os equinos, separados em quatro grupos (G) de seis animais, eram formados por duas fêmeas, três machos castrados e um não castrado. Um constituiu o grupo-controle instrumentado (GI) sem a realização da obstrução intestinal, porém submetidos aos mesmos procedimentos anestésicos e cirúrgicos descritos para os animais dos demais grupos - e os outros três grupos obstruídos. As obstruções intestinais foram realizadas em três, os diferentes segmentos: no duodeno (GII), íleo (GIII) e no cólon maior flexura pélvica (GIV).

Os animais, contidos em brete e após tricotomia e antissepsia da fossa paralombar, foram sedados com acepromazina $1 \%{ }^{5}\left(0,025 \mathrm{mg} \mathrm{kg}^{-1}\right.$, IV), cloridrato de xilazina $2 \%{ }^{6}\left(0,5 \mathrm{mg} \mathrm{kg}^{-1}\right.$, IV) e meperidina $^{7}$ (4mg kg-1, IM). Ato contínuo procedeu-se à anestesia local infiltrativa, utilizando uma associação (1:1) de lidocaína $2 \%{ }^{8}$ e de bupivacaína $0,75 \%$, ambas sem vasoconstritor. Visando mimetizar ao máximo as condições naturais, os animais não foram submetidos a jejum hídrico e alimentar prévios.

Com os animais em estação, realizou-se a laparotomia, fossa paralombar direita para o duodeno e o íleo e esquerda para o cólon maior (flexura pélvica), por meio de incisão da pele de aproximadamente $20 \mathrm{~cm}$ e divulsão romba dos músculos oblíquos, transverso e do peritônio. Em seguida, a cavidade peritonial foi explorada e, ao não se encontrar qualquer anormalidade, identificaram-se os segmentos intestinais; ligaduras feitas com dreno de Penrose $n^{\circ} 3$ foram colocadas ao redor da alça intestinal, aplicadas de modo a não ocluir a irrigação mesentérica, segundo modelo de obstrução descrito por Datt e Usenik (1975). Neste momento os animais receberam $1,5 \mathrm{mg} \mathrm{kg}^{-1}$, IV, de cloridrato de tramadol $^{10}$. Sequencialmente, procedeu-se a sutura simples contínua dos músculos transversos do abdômen e da pele, utilizando-se de vicril $n^{\circ} 2-0$ e náilon $n^{\circ} 4$, respectivamente. As

${ }^{4}$ Omolen Ephos - Purina do Brasil - Paulínea, Brasil

${ }^{5}$ Acepran $1 \%$ - Univet S/A - São Paulo, Brasil.

${ }^{6}$ Virbaxil 2\% - Virbac do Brasil - São Paulo, Brasil.

${ }^{7}$ Dolosal - Cristália Prod. Quim. Farm. Ltda. - Itapira, Brasil.

${ }^{8}$ Lidovet - Laboratório Bravet Ltda. - Rio de Janeiro, Brasil.

${ }^{9}$ Neocaína 0,75\% - Cristália Prod. Quim. Farm. Ltda. - Itapira, Brasil.

${ }^{10}$ Tramal - Cristália Prod. Quim. Farm. Ltda. - Itapira, Brasil. 
obstruções foram mantidas por três horas e, após este período, promoveu-se a reversão das obstruções, tendo como acesso cirúrgico e protocolo anestésico os mesmos utilizados para promovê-las. Os drenos foram então removidos e as cavidades abdominais fechadas de acordo com a técnica descrita por Turner e Mcllwraith (2002).

No pós-operatório, foi instituída terapia antimicrobiana com penicilina benzatina ${ }^{11}$, na dose de $30.000 \mathrm{UIkg}^{-1}$, IM, a cada $48 \mathrm{~h}$, perfazendo três aplicações. Como analgésico e anti-inflamatório, administrou-se flunixim meglumine ${ }^{12}$, na dose de $0,5 \mathrm{mg} \mathrm{kg}^{-1}, \mathrm{IV}$, a cada $24 \mathrm{~h}$, durante dois dias. Curativo da ferida cirúrgica com polivinilpirrolidona-iodo tópica a $1 \%$ foi realizado duas vezes ao dia até a retirada dos pontos no $10^{\circ}$ dia pós-operatório.

Os tempos de colheitas das amostras de líquido peritoneal foram: uma hora antes do início do procedimento cirúrgico (T0 ou basal), T60i, T120i e T180i minutos, correspondente à fase de isquemia ou de obstrução, T60ri, T120ri e T180ri, minutos correspondente à fase de reperfusão imediata ou de desobstrução, e $\mathrm{T} 1^{\circ}$, $\mathrm{T} 3^{\circ}, \mathrm{T}^{\circ} \stackrel{\mathrm{o}}{\text { e }} \mathrm{T} 7^{\circ}$ dias, correspondente à fase de reperfusão tardia ou pós-operatório.

Para colheita do líquido peritoneal, foi realizada a paracentese abdominal segundo a técnica de White (1990), com o auxílio de uma cânula mamária de $60 \mathrm{~mm}$ de comprimento, colhendo-se o líquido em frascos estéreis com e sem EDTA para posterior análise laboratorial. $\mathrm{Na}$ avaliação macroscópica, após a homogeneização das amostras, foram observados a coloração e o grau de turbidez. A coloração foi classificada em amarelo-palha, vermelha e outras (amarelo-ouro, castanha), e o grau de turbidez em límpido, ligeiramente turvo e turvo.

Para a avaliação dos parâmetros bioquímicos, do fósforo inorgânico, pelo método de BasquesLustosa, e das proteínas totais, método do Biureto, foram utilizadas amostras de fluido

\footnotetext{
${ }^{11}$ Pentabiótico Veterinário Reforçado - Fort Dodge Saúde Animal São Paulo, Brasil.
}

${ }^{12}$ Flunixina Injetável - UCB S/A - São Paulo, Brasil. peritoneal colhidas em frascos sem anticoagulante e analisadas com o auxílio de um conjunto de reagentes para diagnósticos ${ }^{13} \mathrm{e}$ posterior leituras espectrofotométricas ${ }^{14}$. Nas amostras colhidas em frascos com EDTA, foram realizadas as análises citológicas. A contagem global de leucócitos foi obtida com o auxílio de um contador automático de $\operatorname{células}^{15}$ e a contagem diferencial foi obtida utilizando-se de esfregaços sanguíneos corados com uma mistura de May-Grunwald, Giemsa e Metanol. Posteriormente, as preparações citoscópicas foram examinadas por microscopia óptica, utilizando microscópio óptico com aumento de 1000x (imersão). A fórmula leucocitária absoluta foi obtida a partir das contagens global e diferencial das células leucocitárias, por uma regra de três direta.

As amostras contendo citrato de sódio (3,8\%) foram utilizadas para dosagem de fibrinogênio pelo método Clauss, e as amostras diluídas em fluoreto de sódio $1 \%$ (1:2) foram utilizadas para a determinação da concentração de lactado, pelo método da lactato oxidase com analisador automático $^{16}$.

Utilizou-se um delineamento experimental inteiramente ao acaso, com quatro grupos e 11 repetições. Quando se constatou significância entre os grupos, dentro de cada momento, aplicou-se $\mathrm{o}$ teste Tukey $(\mathrm{P}<0,05)$ para comparação das médias, por meio do programa estatístico SAS/1999. Para os dados não paramétricos (coloração e turbidez), foi feita uma distribuição de frequências para cada momento de avaliação.

\section{RESULTADOS E DISCUSSÃO}

Os resultados da análise macroscópica, das contagens global e diferencial de leucócitos, com as respectivas médias, desvios-padrão e estatística calculada estão expressos nas Tab. 1 a 3.

\footnotetext{
${ }^{13}$ Labtest - Labtest Diagnóstica S/A - Lagoa Santa, Brasil.

${ }^{14}$ Labquest - Labtest Diagnóstica S/A - Lagoa Santa, Brasil. MG.

${ }^{15}$ AcT-8 Coulter - SG Tecnologia Clínica - São Paulo, Brasil.

${ }^{16}$ Lactímetro YSL 1500 Sport - Yelow Springs, Ohio, EUA.
} 


\section{Di Filippo et al.}

Tabela 1. Variações percentuais da cor e turbidez do líquido peritoneal dos equinos em relação ao momento de colheita

\begin{tabular}{cccccccc}
\hline Momento & $\begin{array}{c}\text { Amarelo- } \\
\text { palha }\end{array}$ & Avermelhado & Amarelo-ouro & Castanho & Límpido & Ligeiramente turvo & Turvo \\
\hline 0 & 100 & 0 & 0 & 0 & 100 & 0 & 0 \\
$60 \mathrm{i}$ & 0 & 100 & 0 & 0 & 0 & 16,67 & 83,33 \\
$120 \mathrm{i}$ & 0 & 100 & 0 & 0 & 0 & 25,00 & 75,00 \\
$180 \mathrm{i}$ & 0 & 100 & 0 & 0 & 0 & 37,50 & 62,50 \\
$60 \mathrm{ri}$ & 0 & 100 & 0 & 0 & 0 & 25,00 & 75,00 \\
$120 \mathrm{ri}$ & 0 & 100 & 0 & 0 & 0 & 20,84 & 79,16 \\
$180 \mathrm{ri}$ & 0 & 70,83 & 29,17 & 0 & 0 & 16,67 & 83,33 \\
$1^{\circ}$ & 0 & 54,16 & 45,84 & 0 & 0 & 16,67 & 83,33 \\
$3^{\circ}$ & 0 & 25,00 & 62,50 & 12,50 & 0 & 12,50 & 87,50 \\
$5^{\circ}$ & 0 & 12,50 & 70,83 & 16,67 & 0 & 8,40 & 91,60 \\
$7^{\circ}$ & 0 & 8,33 & 83,34 & 8,33 & 0 & 8,40 & 91,60 \\
Média geral & 9,09 & 60,98 & 26,51 & 3,42 & 9,09 & 17,05 & 73,84
\end{tabular}

0: basal; 60i-180i: período de isquemia ou de obstrução; 60ri-T180ri: período de reperfusão imediata ou de desobstrução; $1^{0}$ - $7^{0}$ : período de reperfusão tardia ou pós-operatório.

Tabela 2. Média e desvio-padrão da contagem global de leucócitos (x103/ $\mu \mathrm{L})$, neutrófilos segmentados $\left(\mathrm{x} 10^{3} / \mu \mathrm{L}\right)$ e bastonetes $\left(\times 10^{3} / \mu \mathrm{L}\right)$ no líquido peritoneal dos equinos segundo os grupos e tempos

\begin{tabular}{|c|c|c|c|c|c|c|c|c|c|c|c|}
\hline Grupo & 0 & $60 \mathrm{i}$ & $120 \mathrm{i}$ & $180 \mathrm{i}$ & 60ri & $\begin{array}{l}\text { Tempo } \\
\text { 120ri }\end{array}$ & 180ri & $1^{\circ}$ & $3^{\circ}$ & $5^{\circ}$ & $7^{\circ}$ \\
\hline \multicolumn{12}{|c|}{ Leucócitos } \\
\hline \multirow[t]{2}{*}{ I } & $0,80 \pm$ & $0,46 \pm$ & $1,16 \pm$ & $1,26 \pm$ & $3,80 \pm$ & $7,13 \pm$ & $9,40 \pm$ & $70,7 \pm$ & $47,1 \pm$ & $43,0 \pm$ & $43,1 \pm$ \\
\hline & 0,1 & 0,10 & 361 & 51 & 1,08 & 1,65 & $1,85 \mathrm{~B}$ & $14,3 \mathrm{~B}$ & $7,4 \mathrm{~B}$ & $4,5 \mathrm{~B}$ & 16,0 \\
\hline \multirow[t]{2}{*}{ II } & $0,65 \pm$ & $0,64 \pm$ & $1,29 \pm$ & $6,05 \pm$ & $11,7 \pm$ & $16,0 \pm$ & $89,6 \pm$ & $118,0 \pm$ & $171,4 \pm$ & $78,1 \pm$ & $87,3 \pm$ \\
\hline & 0,53 & 0,23 & 931 & 4,57 & 7,7 & 10,6 & $164,4 \mathrm{~A}$ & $61,7 \mathrm{AB}$ & $176,7 \mathrm{~A}$ & $62,5 \mathrm{AB}$ & 92,1 \\
\hline \multirow[t]{2}{*}{ III } & $0,66 \pm$ & $0,70 \pm$ & $1,35 \pm$ & $6,76 \pm$ & $10,0 \pm$ & $13,2 \pm$ & $16,3 \pm$ & $144,3 \pm$ & $145,8 \pm$ & $126,2 \pm$ & $46,5 \pm$ \\
\hline & 0,37 & 0,38 & 900 & 4,44 & 3,3 & 5,2 & $8,4 \mathrm{AB}$ & $106,1 \mathrm{~A}$ & $230,2 \mathrm{~A}$ & $164 \mathrm{~A}$ & 27,4 \\
\hline \multirow[t]{2}{*}{ IV } & $3,70 \pm$ & $3,38 \pm$ & $4,71 \pm$ & $10,7 \pm$ & $16,4 \pm$ & $24,5 \pm$ & $31,3 \pm$ & $79,5 \pm$ & $62,2 \pm$ & $58,0 \pm$ & $68,7 \pm$ \\
\hline & 5,07 & 4,56 & 5,16 & 9,1 & 7,3 & 9,4 & $11,1 \mathrm{AB}$ & $76,9 \mathrm{~B}$ & $23,9 \mathrm{~B}$ & $57,8 \mathrm{AB}$ & 42,8 \\
\hline \multicolumn{12}{|c|}{ Neutrófilo segmentado } \\
\hline \multirow[t]{2}{*}{ I } & $0,55 \pm$ & $0,39 \pm$ & $0,89 \pm$ & $1,11 \pm$ & $3,00 \pm$ & $5,66 \pm$ & $6,17 \pm$ & $59,20 \pm$ & $29,87 \pm$ & $30,74 \pm$ & $35,34 \pm$ \\
\hline & 0,26 & 0,92 & 0,26 & 1,02 & 0,63 & 1,13 & $0,44 \mathrm{~B}$ & $13,59 \mathrm{~B}$ & $3,58 \mathrm{C}$ & $2,73 \mathrm{~B}$ & 12,3 \\
\hline \multirow[t]{2}{*}{ II } & $0,33 \pm$ & $0,40 \pm$ & $1,06 \pm$ & $5,63 \pm$ & $10,72 \pm$ & $14,17 \pm$ & $72,77 \pm$ & $98,39 \pm$ & $135,34 \pm$ & $61,83 \pm$ & $75,41 \pm$ \\
\hline & 0,10 & 0,14 & 0,92 & 4,45 & 7,15 & 9,46 & $13,3 \mathrm{~A}$ & $\begin{array}{l}42,21 \mathrm{~A} \\
\mathrm{~B}\end{array}$ & $129,2 \mathrm{~A}$ & $44,67 \mathrm{AB}$ & 73,5 \\
\hline \multirow[t]{2}{*}{ III } & $0,33 \pm$ & $0,48 \pm$ & $1,19 \pm$ & $6,15 \pm$ & $8,94 \pm$ & $11,42 \pm$ & $13,61 \pm$ & $12,291 \pm$ & $106,44 \pm$ & $99,30 \pm$ & $36,20 \pm$ \\
\hline & 0,10 & 0,25 & 0,92 & 4,32 & 3,20 & 4,67 & $6,92 \mathrm{AB}$ & $4,87 \mathrm{~A}$ & $159,4 \mathrm{AB}$ & $128,1 \mathrm{~A}$ & 24,0 \\
\hline \multirow[t]{2}{*}{ IV } & $2,45 \pm$ & $2,58 \pm$ & $3,65 \pm$ & $9,26 \pm$ & $14,04 \pm$ & $20,87 \pm$ & $24,91 \pm$ & $58,76 \pm$ & $51,34 \pm$ & $48,93 \pm$ & $56,77 \pm$ \\
\hline & 3,76 & 3,42 & 3,81 & 7,88 & 6,28 & 7,53 & $9,37 \mathrm{AB}$ & $9,54 \mathrm{~B}$ & $24,27 \mathrm{BC}$ & $51,16 \mathrm{AB}$ & 32,9 \\
\hline \multicolumn{12}{|c|}{ Neutrófilo bastonete } \\
\hline I & $0,0 \pm 0,0$ & $0,0 \pm 0,0$ & $0,0 \pm 0,0$ & $0,0 \pm 0,0$ & $0,0 \pm 0,0$ & $0,0 \pm 0,0$ & $0,0 \pm 0,0$ & $0,0 \pm 0,0$ & $0,01 \pm 0,0$ & $0,0 \pm 0,0$ & $0,0 \pm 0,0$ \\
\hline II & $0,0 \pm 0,0$ & $0,0 \pm 0,0$ & $0,0 \pm 0,0$ & $0,0 \pm 0,0$ & $0,0 \pm 0,0$ & $0,0 \pm 0,0$ & $0,0 \pm 0,0$ & $0,0 \pm 0,0$ & $0,0 \pm 0,0$ & $0,0 \pm 0,0$ & $0,0 \pm 0,0$ \\
\hline III & $0,0 \pm 0,0$ & $0,0 \pm 0,0$ & $0,0 \pm 0,0$ & $0,0 \pm 0,0$ & $0,0 \pm 0,0$ & $0,0 \pm 0,0$ & $0,0 \pm 0,0$ & $0,0 \pm 0,0$ & $0,0 \pm 0,0$ & $0,0 \pm 0,0$ & $0,0 \pm 0,0$ \\
\hline IV & $0,0 \pm 0,0$ & $0,0 \pm 0,0$ & $0,0 \pm 0,0$ & $0,01 \pm 0,00$ & $0,0 \pm 0,0$ & $0,0 \pm 0,0$ & $0,0 \pm 0,0$ & $0,0 \pm 0,0$ & $0,0 \pm 0,0$ & $0,0 \pm 0,0$ & $0,0 \pm 0,0$ \\
\hline
\end{tabular}

0: basal; 60i-180i: período de isquemia ou de obstrução; 60ri-T180ri: período de reperfusão imediata ou de desobstrução; $1^{\circ}$ - $7^{\mathbf{0}}$ : período de reperfusão tardia ou pós-operatório; GI=controle-instrumentado; GII=obstrução de duodeno; GIII=obstrução de íleo; GIV=obstrução de cólon maior (flexura pélvica).

Médias seguidas por letras distintas, na mesma coluna, diferem entre si pelo teste Tukey $(P<0,05)$ e estabelecem comparação entre os diferentes grupos em cada momento. 


\section{Características celulares e bioquímicas...}

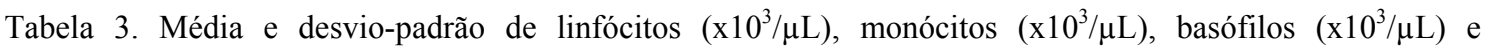
eosinófilos $\left(\times 10^{3} / \mu \mathrm{L}\right)$ no líquido peritoneal dos equinos segundo os grupos e tempos

\begin{tabular}{|c|c|c|c|c|c|c|c|c|c|c|c|}
\hline \multirow{2}{*}{ Grupo } & \multicolumn{11}{|c|}{ Tempo } \\
\hline & 0 & $60 \mathrm{i}$ & $120 \mathrm{i}$ & $180 \mathrm{i}$ & 60ri & 120ri & 180ri & $1^{\circ}$ & $3^{\circ}$ & $5^{\circ}$ & $7^{0}$ \\
\hline \multicolumn{12}{|c|}{ Linfócitos } \\
\hline \multirow[t]{2}{*}{ I } & $0,02 \pm$ & $0,00 \pm$ & $0,01 \pm$ & $0,00 \pm$ & $0,00 \pm$ & $0,01 \pm$ & $0,07 \pm$ & $1,64 \pm$ & $1,34 \pm$ & $0,43 \pm$ & $1,57 \pm$ \\
\hline & 0,01 & 0,00 & 0,0 & 0,00 & 0,01 & 0,02 & 0,05 & 1,27 & $0,61 \mathrm{~B}$ & $0,04 \mathrm{~B}$ & 0,87 \\
\hline \multirow[t]{2}{*}{ II } & $0,05 \pm$ & $0,08 \pm$ & $0,06 \pm$ & $0,06 \pm$ & $0,06 \pm$ & $0,17 \pm$ & $1,28 \pm$ & $2,39 \pm$ & $3,70 \pm$ & $2,58 \pm$ & $1,45 \pm$ \\
\hline & 0,06 & 0,08 & 0,06 & 0,06 & 0,08 & 0,14 & 1,50 & 2,37 & $3,44 \mathrm{AB}$ & $1,78 \mathrm{AB}$ & 1,95 \\
\hline \multirow[t]{2}{*}{ III } & $0,03 \pm$ & $0,04 \pm$ & $0,02 \pm 0$ & $0,07 \pm$ & $0,12 \pm$ & $0,23 \pm$ & $0,26 \pm$ & $4,02 \pm$ & $6,29 \pm$ & $4,97 \pm$ & $1,01 \pm$ \\
\hline & 0,02 & 0,05 & 03 & 0,06 & 0,10 & 0,21 & 0,38 & 1,02 & $1,19 \mathrm{~A}$ & $8,81 \mathrm{~A}$ & 1,01 \\
\hline \multirow[t]{2}{*}{ IV } & $0,12 \pm$ & $0,21 \pm$ & $0,07 \pm$ & $0,12 \pm$ & $0,20 \pm$ & $0,39 \pm$ & $0,42 \pm$ & $1,95 \pm$ & $1,68 \pm$ & $1,81 \pm$ & $1,54 \pm$ \\
\hline & 0,14 & 0,42 & 0,05 & 0,15 & 0,26 & 0,40 & 0,55 & 1,17 & $1,22 \mathrm{~B}$ & $1,76 \mathrm{~B}$ & 1,45 \\
\hline \multicolumn{12}{|c|}{ Monócitos } \\
\hline \multirow[t]{2}{*}{ I } & $0,21 \pm$ & $0,06 \pm$ & $0,25 \pm$ & $0,15 \pm$ & $0,78 \pm$ & $1,45 \pm$ & $3,15 \pm$ & $9,90 \pm$ & $15,35 \pm$ & $11,60 \pm$ & $6,25 \pm$ \\
\hline & 0,11 & 0,00 & 0,97 & 0,05 & 0,04 & 0,54 & 1,35 & 4,61 & $10,11 \mathrm{~B}$ & 6,96 & 2,74 \\
\hline \multirow[t]{2}{*}{ II } & $0,25 \pm$ & $0,12 \pm$ & $0,13 \pm$ & $0,33 \pm$ & $0,91 \pm$ & $1,64 \pm$ & $15,61 \pm$ & $17,20 \pm$ & $29,39 \pm$ & $11,91 \pm$ & $9,99 \pm$ \\
\hline & 0,36 & 0,09 & 0,09 & 0,19 & 0,78 & 1,75 & 29,8 & 9,82 & $45,54 \mathrm{~A}$ & 7,12 & 1,75 \\
\hline \multirow[t]{2}{*}{ III } & $0,29 \pm$ & $0,15 \pm$ & $0,11 \pm$ & $0,48 \pm$ & $0,99 \pm$ & $1,62 \pm$ & $2,41 \pm 1$ & $17,43 \pm$ & $30,62 \pm$ & $20,10 \pm$ & $7,20 \pm$ \\
\hline & 0,31 & 0,09 & 0,10 & 0,37 & 0,38 & 0,72 & 56 & 4,44 & $54,34 \mathrm{~A}$ & 26,35 & 5,27 \\
\hline \multirow[t]{2}{*}{ IV } & $1,11 \pm$ & $0,53 \pm$ & $0,93 \pm$ & $1,27 \pm$ & $2,16 \pm$ & $3,52 \pm$ & $5,60 \pm$ & $18,82 \pm$ & $28,32 \pm$ & $6,85 \pm$ & $10,33 \pm$ \\
\hline & 1,31 & 0,70 & 0,13 & 1,37 & 1,28 & 1,60 & 2,95 & 8,75 & $41,14 \mathrm{AB}$ & 5,47 & 11,1 \\
\hline \multicolumn{12}{|c|}{ Basófilos } \\
\hline \multirow[t]{2}{*}{ I } & $0,0 \pm$ & $0,0 \pm$ & $0,0 \pm$ & $0,0 \pm$ & $0,0 \pm$ & $0,0 \pm$ & $0,0 \pm$ & $0,0 \pm$ & $0,01 \pm$ & $0,00 \pm$ & $0,0 \pm$ \\
\hline & 0,0 & 0,0 & 0,0 & 0,0 & 0,0 & 0,0 & 0,0 & 0,0 & 0,02 & $0,00 \mathrm{~B}$ & 0,0 \\
\hline \multirow[t]{2}{*}{ II } & $0,0 \pm$ & $0,0 \pm$ & $0,0 \pm$ & $0,0 \pm$ & $0,0 \pm$ & $0,0 \pm$ & $0,0 \pm$ & $0,0 \pm$ & $0,0 \pm$ & $0,33 \pm$ & $0,0 \pm$ \\
\hline & 0,0 & 0,0 & 0,0 & 0,0 & 0,0 & 0,0 & 0,0 & 0,0 & 0,0 & $0,81 \mathrm{~A}$ & 0,0 \\
\hline \multirow[t]{2}{*}{ III } & $0,0 \pm$ & $0,0 \pm$ & $0,0 \pm$ & $0,01 \pm$ & $0,0 \pm$ & $0,0 \pm$ & $0,0 \pm$ & $0,0 \pm$ & $0,0 \pm$ & $0,00 \pm$ & $0,0 \pm$ \\
\hline & 0,0 & 0,0 & 0,0 & 0,02 & 0,0 & 0,0 & 0,0 & 0,0 & 0,0 & $0,00 \mathrm{~B}$ & 0,0 \\
\hline \multirow[t]{2}{*}{ IV } & $0,0 \pm$ & $0,0 \pm$ & $0,0 \pm$ & $0,01 \pm$ & $0,0 \pm$ & $0,0 \pm$ & $0,0 \pm$ & $0,0 \pm$ & $0,0 \pm$ & $0,00 \pm$ & $0,0 \pm$ \\
\hline & 0,0 & 0,0 & 0,0 & 0,03 & 0,0 & 0,0 & 0,0 & 0,0 & 0,0 & $0,00 \mathrm{~B}$ & 0,0 \\
\hline \multicolumn{12}{|c|}{ Eosinófilos } \\
\hline \multirow[t]{2}{*}{ I } & $0,00 \pm$ & $0,00 \pm$ & $0,00 \pm$ & $0,00 \pm$ & $0,00 \pm$ & $0,00 \pm$ & $0,00 \pm$ & $0,00 \pm$ & $0,57 \pm$ & $0,30 \pm$ & $0,00 \pm$ \\
\hline & 0,00 & 0,00 & 0,00 & 0,00 & 0,00 & 0,00 & 0,00 & 0,00 & $0,37 \mathrm{~B}$ & $0,23 \mathrm{~B}$ & 0,00 \\
\hline \multirow[t]{2}{*}{ II } & $0,01 \pm$ & $0,02 \pm$ & $0,02 \pm$ & $0,02 \pm$ & $0,02 \pm$ & $0,01 \pm$ & $0,00 \pm$ & $0,00 \pm$ & $2,97 \pm$ & $1,49 \pm$ & $0,50 \pm$ \\
\hline & 0,01 & 0,01 & 0,02 & 0,03 & 0,04 & 0,04 & 0,00 & 0,00 & $5,76 \mathrm{~A}$ & $1,25 \mathrm{AB}$ & 0,66 \\
\hline \multirow[t]{2}{*}{ III } & $0,00 \pm$ & $0,01 \pm$ & $0,00 \pm$ & $0,03 \pm$ & $0,02 \pm$ & $0,00 \pm$ & $0,00 \pm$ & $0,00 \pm$ & $2,44 \pm$ & $1,91 \pm$ & $0,44 \pm$ \\
\hline & 0,00 & 0,01 & 0,14 & 0,06 & 0,05 & 0,00 & 0,00 & 0,00 & $4,82 \mathrm{~A}$ & $1,68 \mathrm{~A}$ & 0,44 \\
\hline \multirow[t]{2}{*}{ IV } & $0,00 \pm$ & $0,04 \pm$ & $0,05 \pm$ & $0,00 \pm$ & $0,06 \pm$ & $0,00 \pm$ & $0,34 \pm$ & $0,00 \pm$ & $0,87 \pm$ & $0,42 \pm$ & $0,00 \pm$ \\
\hline & 0,01 & 0,08 & 0,10 & 0,01 & 0,10 & 0,00 & 0,27 & 0,00 & $0,10 \mathrm{~B}$ & $0,50 \mathrm{~B}$ & 0,00 \\
\hline
\end{tabular}

0: basal; 60i-180i: período de isquemia ou de obstrução; 60ri-T180ri: período de reperfusão imediata ou de desobstrução; $1^{\circ}$ - $7^{0}$ : período de reperfusão tardia ou pós-operatório; $\mathrm{GI}=$ controle-instrumentado; $\mathrm{GII}=\mathrm{obstrução} \mathrm{de} \mathrm{duodeno;} \mathrm{GIII=obstrução} \mathrm{de} \mathrm{íleo;}$ $\mathrm{GIV}=$ obstrução de cólon maior (flexura pélvica). Médias seguidas por letras diferentes, na mesma coluna, diferem entre si pelo teste Tukey $(\mathrm{P}<0,05)$ e estabelecem comparação entre os diferentes grupos em cada momento.

Neste ensaio, as abdominocenteses foram realizadas com cânula mamária, e todas as colheitas foram produtivas e nenhuma enterocentese foi observada. Uma única incisão abdominal era realizada no T0 por meio da qual eram realizadas todas as colheitas subsequentes, atentando unicamente a fatores comuns a qualquer procedimento invasivo, antissepsia local e utilização de luvas e cânulas estéreis. A colheita de líquido peritoneal com agulha 
hipodérmica é, segundo Lopes et al. (1999), menos trabalhosa e menos traumática que a colheita por meio de cânula mamária, entretanto enterocenteses acidentais são frequentemente associadas a esta técnica (Louro et al., 2006).

A penetração de uma agulha em um segmento sadio não distendido de uma alça intestinal é, provavelmente, livre de consequências graves para o animal, pois um orifício pequeno fecha-se rapidamente (Tulleners, 1983). Entretanto, em ensaio realizado por Lopes et al. (1999), dois dos oito animais submetidos à enterocentese acidental desenvolveram, como complicações, peritonite e celulite severa da parede abdominal. O óbito ocorreu no $14^{\mathrm{o}}$ dia pós-operatório no animal que desenvolveu peritonite.

No período que antecedeu à obstrução intestinal (T0), a cor do líquido peritoneal variou de amarelo-palha a incolor, aspecto límpido e ausência de sedimentos, demonstrando a integridade do endotélio vascular e do peritônio (Thomassian, 1996). A contaminação sanguínea, decorrente da laparotomia, provavelmente foi a responsável pelo vermelho observado após as cirurgias (Tab. 1). Nas colheitas seguintes, o líquido adquiriu, gradativamente, coloração amarelada normal sem, contudo, atingir a coloração inicial. Coloração castanha e grande quantidade de sedimentos foram observadas do terceiro ao sétimo dia pós-operatório $\left(\mathrm{T}^{\circ}{ }^{\mathrm{o}}\right.$ a $\left.\mathrm{T} 7^{\circ}\right)$. $\mathrm{O}$ aspecto variou de ligeiramente turvo a turvo, decorrente não apenas da quantidade de proteínas e de células presentes na amostra como, também, da contaminação sanguínea (Louro et al., 2006). Ainda segundo estes autores, um pequeno número de eritrócitos presentes na amostras é capaz de aumentar a turbidez, sem que ocorra o aparecimento da cor avermelhada.

No T0, a média global de leucócitos verificada no líquido peritoneal dos animais de GI, GII, GIII e GIV apresentou-se dentro dos limites de normalidade descritos na literatura (Thomassian, 1996), e assim se mantiveram durante a primeira e a segunda hora de isquemia/obstrução (T60i e T120i). Somente após três horas de reperfusão (T180ri) e no pós-operatório $\left(\mathrm{T} 1^{\circ}, \mathrm{T}^{\circ}{ }^{\circ}\right.$ e $\left.\mathrm{T} 5^{\circ}\right)$ houve aumento significativo do número de leucócitos no líquido peritoneal dos animais do GII e GIII. Segundo Ricketts (1987), o aumento da contagem de células brancas em estágios iniciais de distúrbios intestinais advém principalmente do aumento de polimorfonucleares (PMN). Em casos crônicos, as células mononucleares e os macrófagos aumentam em relação aos polimorfonucleares. A elevação acentuada da leucometria, devido principalmente aos PMN, verificada no líquido peritoneal dos animais do GII e GIII, ocorreu pelo fato de os neutrófilos serem o principal tipo de células que se faz presente nos processos inflamatórios, atuando rápida e efetivamente na defesa celular primária contra os microrganismos, por meio de seus movimentos ameboides rápidos, de sua intensa atividade fagocitária e do conteúdo enzimático, presente nas suas granulações lisossômicas, o qual contém, entre outras, proteínas catiônicas que possuem efeito quimiotático sobre os monócitos e inibem o deslocamento dos neutrófilos e eosinófilos.

Resultados semelhantes foram obtidos por Mendes et al. (1999a) em equinos submetidos à peritonite experimental por meio da inoculação intraperitoneal de Bacteroides fragilis e Escherichia coli. Valores entre 100.000 e 150.000 leucócitos/ $\mu \mathrm{L}$ sugerem, segundo Ricketts (1987) e Thomassian (1996), grave inflamação abdominal com instalação de extensa peritonite, provavelmente séptica. Nesses casos, independente da origem da peritonite, experimental ou natural, os principais sinais clínicos apresentados pelos animais são hipertermia, taquicardia e taquipneia (Mendes et al., 1999b). Nesse ensaio, apesar do aumento significativo da leucometria $\left(\max .171,4 \times 10^{3} / \mu 1\right)$ apresentado pelos animais submetidos à obstrução de segmentos de intestino delgado (GII e GIII), não foram observadas alterações clínicas relevantes que pudessem ser indicativas da presença de processo inflamatório abdominal.

No terceiro dia pós-operatório $\left(\mathrm{T}^{\circ}{ }^{\circ}\right)$, verificou-se eosinofília no líquido peritoneal dos animais do GII e GIII, que ainda se manteve no $\mathrm{T}^{\circ}{ }^{\circ}$ nos animais do GIII. Resultado semelhante foi observado por Mendes et al. (1999a) em equinos com peritonite experimental. Segundo esses autores, isso pode ter sido ocasionado por infestação parasitária, apesar de os animais terem sido vermifugados. A migração de larvas de parasitas pelos tecidos acarreta a liberação de histamina, aumentando, consequentemente, o número de eosinófilos. 
Unicamente no quinto dia pós-operatório $\left(\mathrm{T}^{\circ}{ }^{\circ}\right.$, constatou-se elevação significativa do número de basófilos no líquido peritoneal dos animais do GII, aumento que justifica o papel fisiológico dos basófilos e mastócitos na indução da inflamação, da coagulação e da fibrinólise, por meio das propriedades biológicas dos fatores presentes em seus grânulos e também nas fases mais tardias das reações inflamatórias (Thrall, 2004).

Houve aumento do número de células mononuclares no líquido peritoneal dos animais do GII e GIII somente durante o pós-operatório $\left(\mathrm{T}^{\circ}{ }^{\mathrm{o}}\right.$ e $\left.\mathrm{T} 5^{\circ}\right)$. Aumento do número de macrófagos pode ocorrer sempre que houver necrose tecidual e demanda de fagócitos, ou seja, quadros de necrose, supuração e trauma. Com relação aos linfócitos como estes são células fundamentais na produção de anticorpos humorais e na imunidade celular, é de se esperar que seu número aumente principalmente nas fases mais tardias dos processos inflamatórios, caracterizando a chamada fase linfocítica ou "de cura", como foi descrito por Thrall, 2004.

$\mathrm{Na}$ Tab. 4, são apresentados os valores de proteínas totais, fibrinogênio, fósforo inorgânico e de lactato, com as respectivas médias, desviospadrão e estatística calculada.

Após três horas de reperfusão (T180ri) e durante o pós-operatório $\left(\mathrm{T}^{\circ}{ }^{\circ}\right.$ a $\left.T 7^{\circ}\right)$ houve aumento de proteína total no líquido peritoneal dos animais do GII e GIII. Este aumento deveu-se ao comprometimento da parede intestinal que, por levar a uma alteração vascular, favorece $o$ extravasamento de elementos plasmáticos para o referido fluido (Mendes et al., 1999a). Valores superiores a 2,5g/dL, segundo Faria et al. (1999), indicam lesão vascular progressiva nas vísceras abdominais.

Apesar de mais elevados que os valores fisiológicos $(>0,1 \mathrm{~g} / \mathrm{dL})$, a hiperfibrinogenemia observada no líquido peritoneal dos animais do ensaio não foi significativa. Resposta inflamatória intensa, desencadeada pelo trauma cirúrgico e pela isquemia intestinal, foi considerada por Faria et al. (1999) e Lopes et al. (1999) como responsável pelo aumento dos valores de fibrinogênio em equinos com cólica.

No final do período de isquemia/obstrução (T180i) e início de reperfusão (T60ri), houve aumento de fósforo inorgânico no líquido peritoneal dos animais do GII e GIII, que ainda se manteve até o quinto dia pós-operatório ( $\left(5^{\circ}\right)$ nos animais do GII. O fósforo, que em condições de isquemia intestinal é permeado, inicialmente, das células intestinais danificadas para o lúmen intestinal e posteriormente para o líquido peritoneal, tem seu aumento estreitamente relacionado com a gravidade das lesões. Segundo Arden e Stick (1988), concentrações acima de 3,6mmol/L sugerem a necessidade de ressecção intestinal ou eutanásia, com $77 \%$ e $76 \%$ de sensibilidade e especificidade, respectivamente.

Neste ensaio, com exceção do momento que antecedeu ao procedimento de obstrução intestinal (T0), concentrações de fósforo acima de $3,6 \mathrm{mmol} / \mathrm{L}$ foram achados constantes entre os animais dos grupos obstruídos, entretanto nenhum dos animais foi a óbito ou submetido à ressecção intestinal. Diferenças como estas podem advir das inúmeras técnicas laboratoriais existentes para a determinação da concentração de fósforo inorgânico, como explicaram Moore et al. (1998).

Somente durante a reperfusão imediata (T60ri a T180ri), verificou-se aumento da concentração de lactato no líquido peritoneal dos animais do GII, entretanto os valores obtidos não ultrapassaram 2mmol/L, limite considerado normal por Franklin e Peloso (2006). Ensaio semelhante, realizado por Datt e Usenik (1975), demonstrou que animais submetidos à obstrução de duodeno também foram os únicos a apresentarem aumento de lactado no líquido peritoneal. Segundo esses autores, a alteração deveu-se à isquemia mais intensa sofrida pela alça duodenal em função da pequena extensão intestinal cranial à obstrução, do conteúdo gástrico e da contínua capacidade secretora do estômago. Creditando essas afirmações, neste ensaio, somente os animais do GII apresentaram refluxo gástrico durante o período de obstrução. As células inflamatórias, especialmente os leucócitos, também podem ser as responsáveis pelo aumento de lactato no foco inflamatório, visto que $80 \%$ de toda glicose metabolizada por essas células são convertidas em lactato (HajiMichael et al., 1999). Leucocitose por neutrofilia $(>10.000 \mathrm{cel} / \mu \mathrm{L})$ foi observada no líquido peritoneal desses animais. 
Tabela 4. Média e desvio-padrão da concentração das proteínas totais (g/dL), fibrinogênio (g/dL), fósforo inorgânico $(\mathrm{mg} / \mathrm{dL})$ e lactato $(\mathrm{mmol} / \mathrm{L})$ no líquido peritoneal dos equinos segundo os grupos e tempos

\begin{tabular}{|c|c|c|c|c|c|c|c|c|c|c|c|}
\hline \multirow{2}{*}{ Grupo } & \multicolumn{11}{|c|}{ Tempo } \\
\hline & 0 & $60 \mathrm{i}$ & $120 \mathrm{i}$ & $180 \mathrm{i}$ & 60ri & 120ri & 180ri & $1^{\circ}$ & $3^{-}$ & $5^{\mathrm{o}}$ & $7^{0}$ \\
\hline \multicolumn{12}{|c|}{ Proteínas totais } \\
\hline \multirow[t]{2}{*}{ I } & $0,50 \pm$ & $0,60 \pm$ & $1,03 \pm$ & $1,03 \pm$ & $1,16 \pm$ & $1,40 \pm$ & $1,50 \pm$ & $2,73 \pm$ & $2,53 \pm$ & $2,43 \pm$ & $2,43 \pm$ \\
\hline & 0,44 & 0,49 & 0,93 & 1,04 & 1,24 & 1,14 & $1,35 \mathrm{~B}$ & $0,56 \mathrm{~B}$ & $1,42 \mathrm{~B}$ & $1,53 \mathrm{~B}$ & $1,52 \mathrm{~B}$ \\
\hline \multirow[t]{2}{*}{ II } & $0,85 \pm$ & $1,20 \pm$ & $1,35 \pm$ & $1,61 \pm$ & $2,22 \pm$ & $2,73 \pm$ & $3,95 \pm$ & $5,98 \pm$ & $4,66 \pm$ & $5,01 \pm$ & $5,36 \pm$ \\
\hline & 0,73 & 0,82 & 0,73 & 0,81 & 0,30 & 0,64 & $0,87 \mathrm{~A}$ & $2,07 \mathrm{~A}$ & $1,37 \mathrm{AB}$ & $1,44 \mathrm{~A}$ & $1,91 \mathrm{~A}$ \\
\hline \multirow[t]{2}{*}{ III } & $0,75 \pm$ & $1,53 \pm$ & $1,83 \pm$ & $2,50 \pm$ & $2,91 \pm$ & $3,08 \pm$ & $4,95 \pm$ & $5,03 \pm$ & $5,05 \pm$ & $4,94 \pm$ & $4,20 \pm$ \\
\hline & 0,85 & 1,26 & 1,43 & 1,65 & 1,50 & 2,06 & $2,20 \mathrm{~A}$ & $2,77 \mathrm{~A}$ & $2,30 \mathrm{~A}$ & $2,30 \mathrm{~A}$ & $1,47 \mathrm{AB}$ \\
\hline \multirow[t]{2}{*}{ IV } & $1,20 \pm$ & $1,50 \pm$ & $1,61 \pm$ & $1,86 \pm$ & $2,64 \pm$ & $2,75 \pm$ & $3,05 \pm$ & $4,05 \pm$ & $4,36 \pm$ & $4,35 \pm$ & $4,14 \pm$ \\
\hline & 1,51 & 1,56 & 1,38 & 1,23 & 1,25 & 1,11 & $1,18 \mathrm{AB}$ & $0,80 \mathrm{AB}$ & $0,61 \mathrm{AB}$ & $0,87 \mathrm{AB}$ & $1,73 \mathrm{AB}$ \\
\hline \multicolumn{12}{|c|}{ Fibrinogênio } \\
\hline \multirow[t]{2}{*}{ I } & $0,10 \pm$ & $0,10 \pm$ & $0,10 \pm$ & $0,10 \pm$ & $0,10 \pm$ & $0,08 \pm$ & $0,10 \pm$ & $0,13 \pm$ & $0,10 \pm$ & $0,16 \pm$ & $0,16 \pm$ \\
\hline & 0,00 & 0,00 & 0,00 & 0,00 & 0,00 & 0,03 & 0,00 & 0,10 & 0,00 & 0,05 & 0,05 \\
\hline \multirow[t]{2}{*}{ II } & $0,09 \pm$ & $0,12 \pm$ & $0,18 \pm$ & $0,18 \pm$ & $0,18 \pm$ & $0,15 \pm$ & $0,18 \pm$ & $0,18 \pm$ & $0,11 \pm$ & $0,20 \pm$ & $0,20 \pm$ \\
\hline & 0,06 & 0,06 & 0,07 & 0,07 & 0,04 & 0,05 & 0,07 & 0,04 & 0,04 & 0,10 & 0,12 \\
\hline \multirow[t]{2}{*}{ III } & $0,15 \pm$ & $0,13 \pm$ & $0,11 \pm$ & $0,15 \pm$ & $0,15 \pm$ & $0,15 \pm$ & $0,18 \pm$ & $0,15 \pm$ & $0,18 \pm$ & $0,20 \pm$ & $0,20 \pm$ \\
\hline & 0,05 & 0,05 & 0,05 & 0,05 & 0,05 & 0,05 & 0,07 & 0,05 & 0,05 & 0,10 & 0,06 \\
\hline \multirow[t]{2}{*}{ IV } & $0,09 \pm$ & $0,15 \pm$ & $0,11 \pm$ & $0,16 \pm$ & $0,13 \pm$ & $0,13 \pm$ & $0,16 \pm$ & $0,15 \pm$ & $0,15 \pm$ & $0,18 \pm$ & $0,16 \pm$ \\
\hline & 0,02 & 0,12 & 0,04 & 0,08 & 0,05 & 0,05 & 0,12 & 0,05 & 0,05 & 0,07 & 0,08 \\
\hline \multicolumn{12}{|c|}{ Fósforo inorgânico } \\
\hline \multirow[t]{2}{*}{ I } & $1,56 \pm$ & $2,06 \pm$ & $2,46 \pm$ & $1,93 \pm$ & $2,36 \pm$ & $2,60 \pm$ & $2,86 \pm$ & $2,93 \pm$ & $2,96 \pm$ & $2,53 \pm$ & $3,06 \pm$ \\
\hline & 0,64 & 0,71 & 1,13 & $0,72 \mathrm{~B}$ & $0,89 \mathrm{~B}$ & $1,58 \mathrm{~B}$ & $0,65 \mathrm{~B}$ & $0,73 \mathrm{~B}$ & $0,75 \mathrm{~B}$ & $0,74 \mathrm{~B}$ & 0,98 \\
\hline \multirow[t]{2}{*}{ II } & $2,70 \pm$ & $3,73 \pm$ & $3,71 \pm$ & $4,30 \pm$ & $5,11 \pm$ & $4,88 \pm$ & $4,88 \pm$ & $4,71 \pm$ & $4,80 \pm$ & $4,48 \pm$ & $4,68 \pm$ \\
\hline & 0,70 & 1,51 & 1,07 & $1,32 \mathrm{~A}$ & $1,31 \mathrm{~A}$ & $1,00 \mathrm{~A}$ & $0,83 \mathrm{~A}$ & $0,86 \mathrm{~A}$ & 0,77A & $0,71 \mathrm{~A}$ & 0,92 \\
\hline \multirow[t]{2}{*}{ III } & $2,15 \pm$ & $2,56 \pm$ & $2,61 \pm$ & $4,08 \pm$ & $4,88 \pm$ & $3,58 \pm$ & $3,48 \pm$ & $3,03 \pm$ & $3,46 \pm$ & $3,06 \pm$ & $3,73 \pm$ \\
\hline & 0,61 & 0,67 & 0,51 & $0,98 \mathrm{~A}$ & $1,06 \mathrm{~A}$ & $0,79 \mathrm{AB}$ & $0,74 \mathrm{AB}$ & $0,62 \mathrm{AB}$ & $0,87 \mathrm{AB}$ & $1,09 \mathrm{AB}$ & 0,63 \\
\hline \multirow[t]{2}{*}{ IV } & $3,10 \pm$ & $3,46 \pm$ & $3,58 \pm$ & $2,96 \pm$ & $3,45 \pm$ & $4,26 \pm$ & $4,20 \pm$ & $2,95 \pm$ & $3,16 \pm$ & $3,43 \pm$ & $3,10 \pm$ \\
\hline & 1,57 & 1,98 & 1,64 & $1,30 \mathrm{AB}$ & $1,98 \mathrm{AB}$ & $1,28 \mathrm{AB}$ & $1,43 \mathrm{AB}$ & $1,30 \mathrm{AB}$ & $1,30 \mathrm{AB}$ & $1,14 \mathrm{AB}$ & 1,55 \\
\hline \multicolumn{12}{|c|}{ Lactato } \\
\hline \multirow[t]{2}{*}{ I } & $0,27 \pm$ & $0,38 \pm$ & $0,53 \pm$ & $0,62 \pm$ & $0,61 \pm$ & $0,61 \pm$ & $0,52 \pm$ & $0,58 \pm$ & $0,61 \pm$ & $0,70 \pm$ & $0,54 \pm$ \\
\hline & 0,35 & 0,54 & 0,80 & 1,07 & $1,37 \mathrm{~B}$ & $1,68 \mathrm{~B}$ & $2,02 \mathrm{~B}$ & 2,31 & 2,62 & 2,92 & 3,28 \\
\hline \multirow[t]{2}{*}{ II } & $0,32 \pm$ & $0,44 \pm$ & $0,48 \pm$ & $0,88 \pm$ & $1,33 \pm$ & $1,14 \pm$ & $1,27 \pm$ & $1,05 \pm$ & $0,75 \pm$ & $0,60 \pm$ & $0,53 \pm$ \\
\hline & 0,58 & 0,66 & 0,88 & 1,12 & $1,29 \mathrm{~A}$ & $1,61 \mathrm{~A}$ & $1,84 \mathrm{~A}$ & 2,18 & 2,59 & 2,96 & 3,30 \\
\hline \multirow[t]{2}{*}{ III } & $0,25 \pm$ & $0,55 \pm$ & $0,51 \pm$ & $0,58 \pm$ & $0,87 \pm$ & $0,96 \pm$ & $0,98 \pm$ & $0,85 \pm$ & $0,72 \pm$ & $0,55 \pm$ & $0,48 \pm$ \\
\hline & 0,87 &, 87 & 1,05 & 1,26 & $1,43 \mathrm{AB}$ & $1,70 \mathrm{AB}$ & $2,02 \mathrm{AB}$ & 2,30 & 2,65 & 2,98 & 3,31 \\
\hline \multirow[t]{2}{*}{ IV } & $0,19 \pm$ & $0,32 \pm$ & $0,39 \pm$ & $0,46 \pm$ & $0,70 \pm$ & $0,81 \pm$ & $0,84 \pm$ & $0,61 \pm$ & $0,38 \pm$ & $0,62 \pm$ & $0,42 \pm$ \\
\hline & 1,20 & 1,21 & 1,31 & 1,47 & $1,61 \mathrm{~B}$ & $1,81 \mathrm{AB}$ & $2,0 \mathrm{AB}$ & 2,46 & 2,80 & 3,02 & 3,37 \\
\hline
\end{tabular}

0: basal; 60i-180i: período de isquemia ou de obstrução; 60ri-T180ri: período de reperfusão imediata ou de desobstrução; $1^{0}$ - $7^{0}$ : período de reperfusão tardia ou pós-operatório; GI=controle-instrumentado; GII=obstrução de duodeno; GIII=obstrução de íleo; GIV=obstrução de cólon maior (flexura pélvica).

Médias seguidas por letras distintas, na mesma coluna, diferem entre si pelo teste Tukey $(\mathrm{P}<0,05)$ e estabelecem comparação entre os diferentes grupos em cada momento.

\section{CONCLUSÕES}

A técnica de abdominocentese com cânula mamária mostrou-se prática, eficiente e segura. O modelo de obstrução intestinal utilizado desencadeou resposta inflamatória mais intensa na cavidade peritoneal dos animais submetidos à obstrução de duodeno e íleo. Entretanto, as alterações laboratoriais não foram associadas a sinais clínicos indicativos da presença de processo inflamatório abdominal e não permitiram a identificação do segmento intestinal 
obstruído visto que ocorreram somente após a desobstrução. A análise seriada do líquido peritoneal auxilia no acompanhamento da evolução do processo de cura, porém não deve ser utilizada isoladamente na elaboração do diagnóstico e prognóstico de complicações no pós-operatório de equinos com cólica.

\section{AGRADECIMENTO}

À Fundação de Amparo à Pesquisa do Estado de São Paulo (Fapesp) pelo financiamento integral desta pesquisa (Proc. $\mathrm{n}^{\circ}$ 05/58712-0 e $\mathrm{n}^{\circ}$ 06/55377-8).

\section{REFERÊNCIAS BIBLIOGRÁFICAS}

ARDEN, W.A.; STICK, J.A. Serum and peritoneal fluid phosphate concentrations as predictors of major intestinal injury associated with equine colic. J. Am. Vet. Med. Assoc., v.193, p.927-930, 1988.

DATT, S.C.; USENIK, E.A. Intestinal obstruction in the horse. Physical signs and blood chemistry. Cornell Vet., v.65, p.152-172, 1975.

FARIA, E.P.; MARQUES JR., A.P.; ALVES, G.E.S. Características celulares e bioquímicas do líquido peritoneal de equinos submetidos à peritonite experimental. Arq. Bras. Med. Vet. Zootec., v.51, p.335-344, 1999.

FRANKLIN, R.P.; PELOSO, J.G. Review of the clinical use of lactate. In: ANNUAL CONVENCTION OF THE AMERICAN ASSOCIATION OF EQUINE PRACTIONERS, 52., 2006. Ithaca. Proceedings... Ithaca: International Veterinary Information Service, 2006. p. 142 .

HAJI-MICHAEL， P.G.; LADRIERE， L.; SENER, A. et al. Leukocyte glycolysis and lactate output in animal sepsis and ex human blood. Metabolism, v.48, p.779-785, 1999.

LOPES, M.A.F.; DEARO, A.C.O.; BIONDO, A.W. et al. Exame do fluido peritoneal e hemograma de equinos submetidos à laparotomia e infusão intraperitoneal de carboximetilcelulose. Cienc. Rural, v.29, p.79-85, 1999.
LOURO, M.F.C.; DIAS, R.V.C.; SOTOBLANCO, B. Avaliação do fluido peritoneal de asininos. Arq. Bras. Med. Vet. Zootec., v.58, p.955-958, 2006.

MENDES, L.C.N.; MARQUES, L.C.; BECHARA, G.H. et al. Experimental peritonitis in horses: peritoneal fluid composition. Arq. Bras. Med. Vet. Zootec., v.5, p.493-497, 1999a.

MENDES, L.C.N.; MARQUES, L.C.; SCHOCKEN-ITURRINO, R.P. et al. Clinical aspects of experimental peritonitis in horses. Cienc. Rural, v.29, p.493-497, 1999b.

MOORE, R.M.; MUIR, W.W.; RUSH, B.R. Systemic and colonic venous plasma biochemical alterations in horses during lowflow ischemia and reperfusion of the large colon. Can. J. Vet. Res., v.62, p.14-20, 1998.

RICKETTS, S.W. Peritonitis In: ROBINSON, N.E. Current therapy in equine medicine. 2.ed. London: W.B. Saunders, 1987. p.79-81.

THOMASSIAN, A. Enfermidade dos cavalos. 3.ed. São Paulo: Varela, 1996. 463p.

THRALL, M.A. Veterinary hematological and clinical chemistry. Philadelphia: Lippincott Willians \& Wilkins, 2004. 518p.

TULLENERS, E.P. Complications of abdominocentesis in the horse. J. Am. Vet. Med. Assoc., v.182, p.232-234, 1983.

TURNER, A.S.; McILWRAITH, C.W. Laparotomia do flanco e exploração abdominal. In: . Técnicas cirúrgicas em animais de grande porte. São Paulo: Roca, 2002. p.237-242.

WHITE, N.A. Determining the diagnosis and prognosis of the acute abdominal. In: WHITE, N.A. The equine acute abdomen. Philadelphia: Lea \& Fabiger, 1990. p.101-140. 